\title{
Quantum dots-based multiplexed immunohistochemistry of protein expression in human prostate cancer cells
}

\author{
C. Shi, ${ }^{1,2}$ G. Zhou, ${ }^{1}$ Y. Zhu, ${ }^{1,2}$ Y. Su, ${ }^{2}$ T. Cheng, ${ }^{2}$ H.E. Zhau, ${ }^{1}$ L.W.K. Chung ${ }^{1}$ \\ ${ }^{1}$ Molecular Urology and Therapeutics Program, Department of Urology and Winship Cancer Institute, \\ Emory University School of Medicine, Atlanta, GA USA; ${ }^{2}$ Chongqing Engineering \& Technology Center for \\ Nanomedicine, International Cooperation Center for Excellence of Nanomedicine, State Key Laboratory \\ of Trauma, Burns and Combined Injury, Institute of Combined Injury, Third Military Medical University, \\ Chongqing, China
}

(C)2008 European Journal of Histochemistry

Semiconductor quantum dots (QDs) are bright fluorescent nanoparticles that have been successfully used for the detection of biomarker expression in cells. The objective of the present study is to use this technology in a multiplexing manner to determine at a single cell level the expression of a cell-specific bio-marker, prostate-specific antigen (PSA) expressed by human prostate cancer LNCaP and ARCaP cell lines. Here we compared the sensitivity of immunohistochemistry (IHC) and QD-based detection of AR and PSA expression in these cell lines. Further, we conducted multiplexing QD-based detection of PSA and androgen receptor (AR) expression in LNCaP cells subjecting to androgen (R1881) stimulation. The involvement of AR in PSA regulation in LNCaP cells, at a single cell level, was confirmed by the co-incubation of LNCaP cells in the presence of both R1881 and its receptor antagonist, bicalutamide (Casodex). We showed here the superior quality of QDs, in comparison to IHC, for the detection of AR and PSA in cultured LNCaP and ARCaP cells. Multiplexing QDs technique can be used to detect simultaneously AR and PSA expression induced by R1881 which promoted AR translocation from its cytosolic to the nuclear compartment. We observed AR antagonist, bicalutamide, inhibited AR nuclear translocation and PSA, but not AR expression in LNCaP cells.

Key words: Multiplexed imaging, quantum dots, prostate cancer.

Correspondence: Haiyen E. Zhau, or Chunmeng Shi,

1365B Clifton Road, Molecular Urology and Therapeutics Program, Department of Urology and Winship Cancer Institute, Emory University School of Medicine,

Atlanta, GA 30322, USA

E-mail: hzhau@emory.edu

shicm1010@yahoo.com.cn

Paper accepted on May 14, 2008

European Journal of Histochemistry

2008; vol. 52 issue 2 (Apr-Jun): 127-134
I mmunohistochemical detection of gene expression has been a widely used technique in cellular and molecular biologic studies. Recent advances in semiconductor quantum dot (QD) chemistry provided an alternative approach to detect gene expression in cells with high degree of sensitivity and specificity (Alivisatos et al., 2005; Pinaud et al., 2006). QD nanoparticles tagged with antibody (Chan and Nie, 1998), proteins or peptides (Zhou et al., 2007), enzyme substrates and organic molecules ((Wu et al., 2003) have been successfully used in the detection of gene expression (Alivisatos et al., 2005) and movement of cells (Michalet et al., 2005) and the delivery of drugs (Yezhelyev et al., 2006) in biologic systems. Because of the sensitivity of fluorescent $Q D$, their photostability and tunable nature (a broad-spectrum of light excitation of $Q D$ can result in the emission of multiple sharp and narrow spectra representative of $Q D$ of different sizes and chemical composition (Smith et al., 2006) for the detection of biomolecules and cells in an organism, QD have become the preferred substrates over organic dyes for tagging cells or cellular components in biologic studies (Michalet et al., 2005; West et al., 2003). In several recent reports (Yezhelyev et al., 2006; Chan et al., 2005), QD have been employed in IHC for the detection of gene expression and localization of key regulatory molecules in cells and tissues. The present study focused on employing the distinct advantages of $Q D$, including its sensitivity and multiplexing capability, to simultaneously detect androgen receptor (AR) and its target gene, prostate-specific antigen (PSA) expression in human prostate cancer $\mathrm{LNCaP}$ and ARCaP cells. We compared IHC and QD-based methods in the detection of $A R$ and PSA in cells with either high ( $L N C a P$ ) or low (ARCaP) levels of expression of $A R$ and PSA. We also employed a multiplexing QD-based technique to detect and quantify $A R$ and PSA simultaneously with cells 
either stimulated by androgen (R1881) or in the presence of both R1881 and its antagonist, bicalutamide (Casodex) to assess the involvement of $A R$ in PSA expression. In sum, we validated that QDbased gene expression methods are superior to IHC and can be used to detect gene expression in a single cell in a multiplexing manner.

\section{Materials and Methods}

\section{Antibodies}

Quantum dot secondary antibody conjugates were from Invitrogen, (Carlsbad, CA) including QD655nm, QD-605nm, and QD-565nm anti-mouse IgGs. Monoclonal antibodies were anti-human prostate specific antigen (PSA) and anti-human androgen receptor (AR) from Santa Cruz Biotechnology (Santa Cruz, CA) and anti-human elongation factor- 1 alpha (EF-1 $\alpha$ ) from Upstate (Billerica, MA). Androgen analog R1881 and fetal bovine serum (FBS) were from Sigma (St. Louis, MO). Androgen antagonist, bicalutamide (Casodex) was obtained from AstraZeneca (Los Angeles, CA). Tissue culture $T$ medium was purchased from Life Technologies (Rockville, MD).

\section{Cell lines and cell culture}

Human prostate cancer cell lines LNCaP and ARCaP were cultured in 8-well chamber slides (Nalge Nunc International, Rochester, NY) in Tmedium supplemented with $5 \%$ FBS and $1 \%$ penicillin/streptomycin as described previously (Gleave, et al., 1991). The cells were maintained at $37^{\circ} \mathrm{C}$ in $5 \% \mathrm{CO}_{2}$ and $95 \%$ air. After culture in T-medium with 5\% FBS for 24 hours, the culture medium was replaced with phenol red free RPMI 1640 with or without $5 \mathrm{nM}$ R1881 in the presence or absence of its antagonist 10 uM Casodex for 48 hours. The cells were rinsed briefly with cold PBS and fixed in $-20^{\circ} \mathrm{C}$ acetone for $10 \mathrm{~min}$ and were used for IHC or QD staining.

\section{Immunostaining with quantum dot probes}

Acetone fixed cells were incubated with a blocking buffer containing $2 \% B S A / 5 \%$ goat serum/PBS for $60 \mathrm{~min}$ at room temperature and were incubated with primary antibodies for one hour at room temperature. The antibody dilutions were PSA (1:50), AR (1:100), and EF-1 $\alpha$ (1:100). After rinsing 3 times with PBS, QD-IgGs (20 nM) were applied to the slides and incubated overnight at $4^{\circ} \mathrm{C}$ or 2 hrs at room temperature. For multiplexed imaging, the three proteins were stained separately and concurrently. After the QD staining, cells were counter-stained with DAPI $(20 \mathrm{ng} / \mathrm{mL})$ for cell nuclei. For conventional IHC staining, the secondary antibodies were replaced by HRP labeled antimouse IgG using hydrogen peroxide as substrate and diaminobenzadine as chromogen, followed by hematoxylin nuclear counterstain.

\section{Imaging and spectroscopy}

True-color fluorescence images were obtained with an inverted Olympus IX-70 microscope and a digital Nikon D70 color camera. Broad-band excitation in the near- $U V$ range $(330-385 \mathrm{~nm})$ was provided by a $100 \mathrm{~W}$ mercury lamp. A longpass dichroic filter (DM 400, Chroma Technologies, Brattleboro, VT) was used to reject the scattered light and to pass the Stokes-shifted fluorescence signals. All images were captured using a $100 \mathrm{X}$ oil objective lens unless otherwise specified. Fluorescence spectra of the QD labeled single cells were collected using a spectrometer (Princeton Instruments, Princeton, NJ) coupled with WinSpec 32 software. For each sample, 40-50 cells were randomly picked for spectral analyses.

\section{Western blot}

Protein was extracted from cell pellets with a lysis buffer $[50 \mathrm{mmol} / \mathrm{L}$ Tris $(\mathrm{pH} 8.0), 150 \mathrm{mmol} / \mathrm{L}$ $\mathrm{NaCl}, 0.02 \% \mathrm{NaN}_{3}, 0.1 \%$ SDS, $1 \% \mathrm{NP} 40,0.5 \%$ sodium deoxycholate, and $1 \mathrm{mmol} / \mathrm{L}$ phenylmethylsulfonyl fluoride] in the presence of protease inhibitor cocktail (Roche Applied Science, Indianapolis, IN). Samples containing equal amounts of protein $(30 \mu \mathrm{g})$ were electrophoresed on Tris-Glycine gels (Invitrogen) and transferred to nitrocellulose membranes. After blocking with Tween-tris buffered saline (T-TBS) containing 5\% nonfat milk powder, membranes were incubated separately and sequentially with mouse monoclonal antibodies against either PSA or AR at $4^{\circ} \mathrm{C}$ overnight. After washing with T-TBS, the membranes were incubated with corresponding secondary antibodies, which were conjugated with horseradish peroxidase (Santa Cruz Biotechnology). The blots were stripped by a standard protocol used in our laboratory. In brief, the membranes were placed in a heated $\left(50^{\circ} \mathrm{C}\right)$ stripping buffer ( $2 \%$ SDS, 100 $\mathrm{mM} \beta$ - mercaptoethanol and $50 \mathrm{mM}$ Tris, $\mathrm{pH}$ 6.8) 
for 15-30 min with gentle shaking. The membranes were rinsed with TBS several times and the blots were ready for re-blot with anti- EF-l $\alpha$ antibody (1:1000 dilution). Immunoreactive bands were visualized with enhanced chemiluminescence (Amersham Pharmacia Biotech, Little Chalfont, United Kingdom) using previously established protocols (Huang et al., 2006; Nomura et al., 2006; Wu et al., 2007).

\section{Deconvolution of composite spectra and quantita- tive analysis}

The photostability and the narrow and Gaussianshaped emission spectra of QDs make it possible to use QDs for absolute quantification of targets and it has been documented that there is a linear relationship between the measured fluorescence intensity and the number of stained QDs (Arya et al., 2005). Based on this assumption, the Gaussian function is chosen to represent each peak in the spectra. The Gaussian function is represented as: $f(x)=\alpha e^{-(x-b) 2 / c^{2}}$ where $a, b$, and $c$ are real constants and $a>0, c>0$; the improper integrals over the whole real line can be evaluated exactly: $\int_{-\infty}^{+\infty} F(x)=$ $\sqrt{\pi}$ ac. Each spectrum can be represented as a sum of $n$ peaks: spectrum $=\sum_{l=1}^{n}$ peak. A linear combination of QD spectra was used to fit the composite photoluminescence signal for each sample. Thus, the composite signal can be calculated as: $I_{\text {total }}(\lambda)=\sum_{I=1}^{n} I$ $(\lambda)=\sqrt{\pi} \sum_{l=1}^{n} a_{i}, c_{i}$ where $a_{i}, c_{i}$ are the parameters in the Gaussian function obtained from fitting the ith peak in the spectrum, $I_{\text {total }}(\lambda)$ is the composite signal (corrected for background signal). The area ratio between ith and jth peaks can be easily obtained by $r=a_{i} c_{i} / a_{i c}$. In this work, we will concentrate on the QD samples $(565,605$, and $655 \mathrm{~nm}$ emission maxima). The $565 \mathrm{~nm}$ peak will be used as the reference. A software package was developed in Matlab that can fit QD spectra with a minimum of 1 to a maximum of 8 peaks for a single spectrum or multiple spectra in a set. The areas under each fitted peak and the ratios of peaks at $605 \mathrm{~nm}$ to $565 \mathrm{~nm}$ and $655 \mathrm{~nm}$ to $565 \mathrm{~nm}$ are also given. For quantitative analysis, the relative expression level of the target gene was calculated as the ratio of the fluorescence intensity of the target gene to the intensity of the control gene. Statistical analysis was performed by Student's t test. The significant level $\alpha=0.05$ was used in the study.

\section{Results}

\section{Optical imaging of low-abundant genes in prostate cancer cells using quantum dots}

We determined the sensitivity of QDs-antibody conjugates in the detection of low abundant $A R$ and PSA in ARCaP cells. As shown in Figure $1 A$, clear fluorescence signals for both AR and PSA proteins were detected in ARCaP cells. PSA was found to be predominately cytoplasmic. AR was found to be present in both cytoplasmic and nuclear compartments. In a parallel study, however, the presence of $A R$ and PSA in ARCaP cells was less apparent when stained by IHC using the same primary antibodies (Figure 1A). One other advantage of QDbased IHC is that under these assay conditions, the fluorescent signal intensity was stable for several hours. These results indicate that the QDs-combined IHC method can detect the expression of genes of low abundance which cannot be clearly discerned using the conventional IHC methods.

\section{Imaging of ligand-induced androgen receptor translocation and PSA expression in LNCaP cells}

Since QD staining proved to be more sensitive than conventional IHC in detecting low-abundant genes, we next analyzed the relationship between AR nuclear translocation and PSA expression in androgen-responsive LNCaP cells. After $24 \mathrm{~h}$ attachment to the plastic dishes, replaced with phenol red free RPMI 1640 medium in the absence of serum, cells were exposed to an androgen analog, R1881, as previously described (Mizokami et al., 2000). As shown in Figure $1 B, A R$ nuclear translocation was clearly visible in LNCaP cells after treatment with R1881 for 48 hours. In control cells without R1881 treatment, AR staining was mostly in the cell cytoplasm. Since PSA expression is known to be under regulation by liganded $A R$, we next examined PSA expression in LNCaP cells under stimulation by R1881 in the presence of an androgen antagonist, Casodex. Results showed that up-regulation of PSA expression in LNCaP cells by R1881 was blocked by Casodex (Figure 1B). These results were further confirmed by Western blot analysis (Figure $1 \mathrm{C}$ ), suggesting QD-antibody conjugates are suitable for the detection of gene expression in prostate cancer cells. These observations are in agreement with our previously published reports where antiandrogen such as Casodex or flutamide antagonized PSA expression in 
LNCaP cells stimulated by androgen (Thalmann et al., 1996; Kiyama et al., 2003; Yeung et al., 2000).

\section{Multiplexed quantum dots imaging of gene expression in a single cell}

Since LNCaP cells express both AR and PSA, we explored the possibility of using this cell line as target for androgen-induced responses by monitoring
AR and PSA simultaneously. Based on the fluorescent characteristics of quantum dots, this makes it an ideal candidate for multiplexing AR and PSA in LNCaP cells. We performed multiplexed staining of $A R, P S A$ and EF-l $\alpha$, an internal standard, in LNCaP cells subjected to R1881 treatment either in the presence or absence of an androgen antagonist, Casodex. Figure 2A showed that multiplexed LNCaP

A

AR
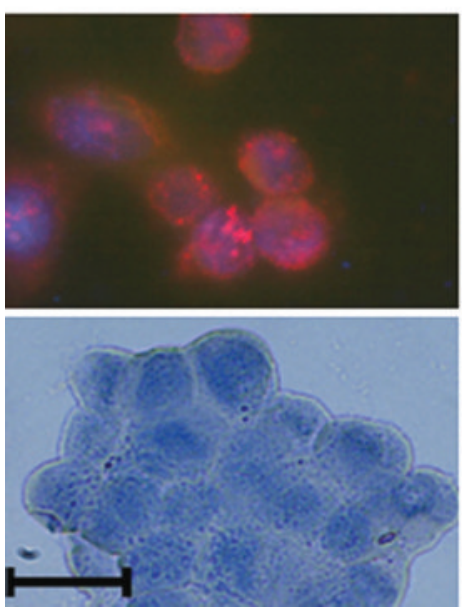

PSA
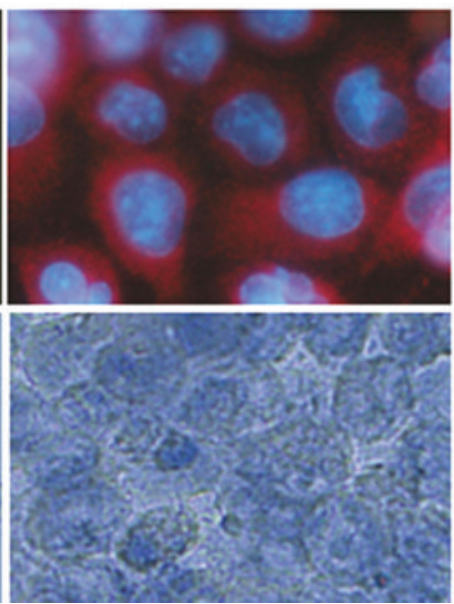

QD-based IHC

Conventional IHC

B

Control
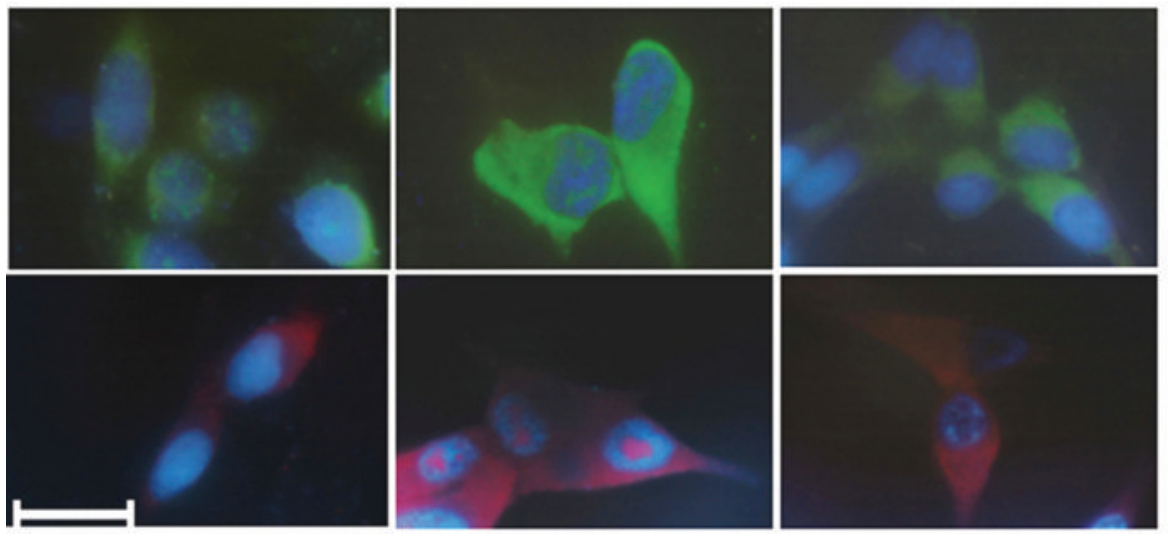

C

Control R1881 R1881+Casodex
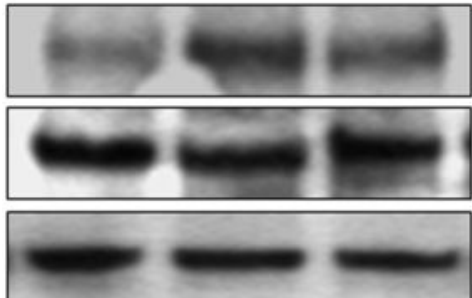

Control R1881 R1881+Casodex

Nuclear AR

Cytoplasm AR

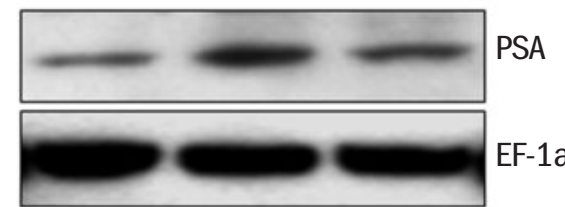

EF-1a
PSA

AR

-1a
Figure 1. Optical imaging of protein markers of prostate cancer cells using quantum dots combined with immunohistochemical staining.

(A) Comparative study of the detection sensitivity of AR and PSA in ARCaP cells between conventional IHC and QD-based IHC. QD605 and QD655 were used to label PSA and AR, respectively. Bar, 20 um.

(B) Optical imaging using QD-based IHC showed that AR nuclear translocation and PSA expression in LNCaP cells was stimulated by androgen analog, R1881. Casodex, an androgen antagonist, blocked the stimulation of PSA expression induced by R1881. QD605 was used to label PSA and QD655 was used to label AR. Bar, 20 um.

(C) Western blot analysis confirmed the AR nuclear translocation and PSA expression in R1881-treated LNCaP cells. 
cells can be imaged quantitatively, at a single cell level, for the expression of all three genes, AR, PSA and EF- $1 \alpha$, using QDs with different emission spectra. We observed AR nuclear translocation in LNCaP cells after R1881 treatment. In order to perform the quantitative analysis, a software program was developed to monitor the gene expression based on fluorescence intensity of the spectrum for the corresponding marker gene. The spectrum files for single cells were obtained using the spectrometer under the fluorescence microscope and the data were processed using the WinSpec 32 program (see
Materials and Methods). As shown in Figure 2B, the fitting spectrum curve generated from the software program matched very well the spectrum curve generated from the real data. We next performed quantitative analysis based upon the ratios of each gene expression to that of the control gene. As shown in Figure 2C, R1881 treatment increased PSA expression in LNCaP cells, but there was no significant increase in the expression of total AR. In the presence of an androgen antagonist, Casodex, it blocked the ability of AR to induce PSA expression without affecting $A R$ expression at the single cell level.

A

Control

R1881

R1881+Casodex
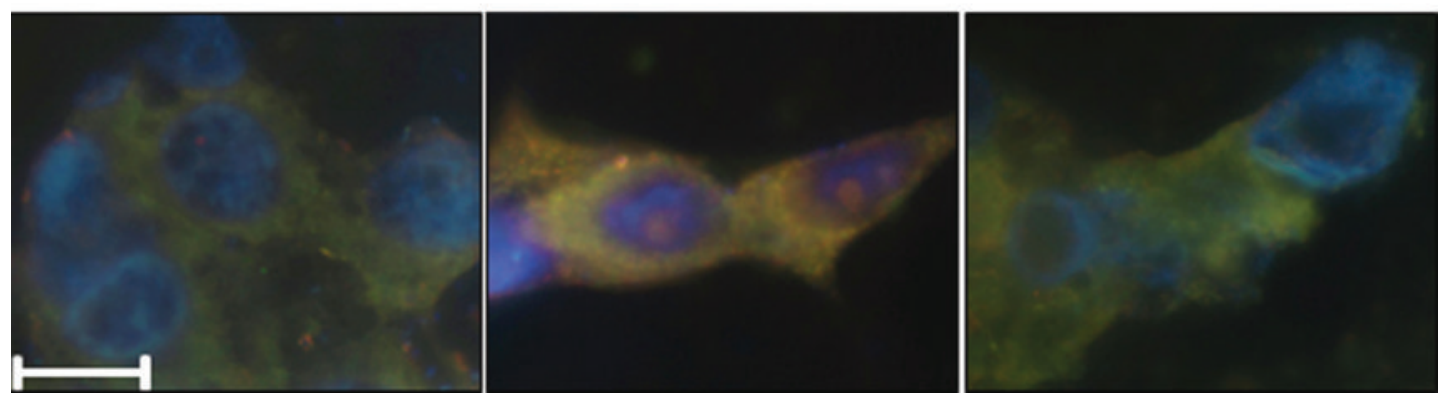

B
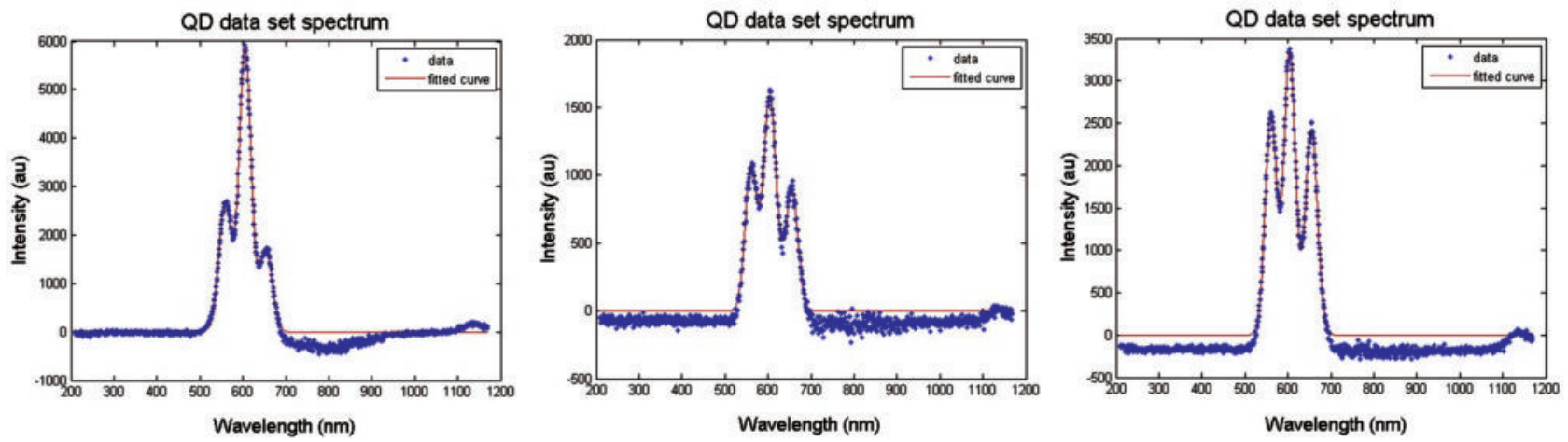

C

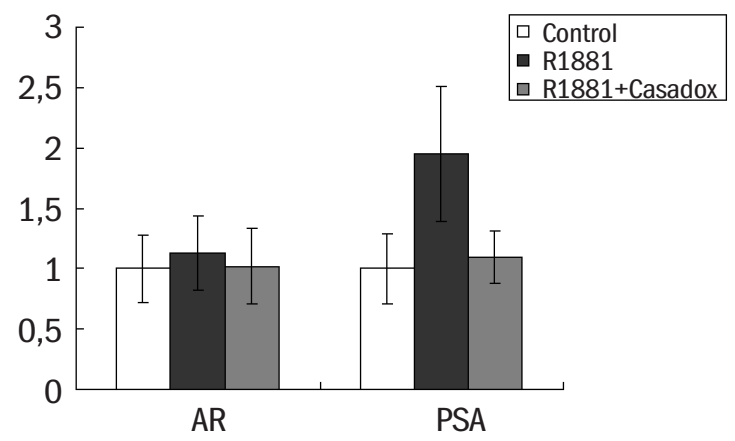

Figure 2. Multiplexed imaging and profiling of protein markers in LNCaP cells. (A) Triple stain of AR, PSA and EF-1 $\alpha$ in single LNCaP cells using QD with QD655, QD605 and QD565, respectively. QD655 (red) was used to label AR, QD605 (orange) was used to label PSA and QD565 (green) was used to label EF-1 $\alpha$. Bar, 20 um. (B) Spectra analysis of the gene expression by the spectrometer. A software program was developed in Matlab that can fit the QD spectra with different peaks from the spectrometer. (C) Quantitative analysis of target gene expression was based on the ratio of the fluorescence intensity of target gene to control gene, EF-1 $\alpha$. indicates the significant difference of PSA expression between R1881 treatment group and control group. 


\section{Discussion}

The present study demonstrated for the first time the use of QD multiplexing technology for the detection of the expression of an androgen target gene, PSA, in a human prostate epithelial cell line, LNCaP, under the influence of androgen and its antagonist, Casodex. We monitored AR and PSA simultaneously with a house-keeping gene, EF-l $\alpha$, as an internal standard. We found that $Q D$ immunostaining of gene expression is superior to conventional IHC with respect to its sensitivity. Though ARCaP cells express low levels of $A R$ and PSA, these markers can be detected readily by QDbased but not chromogen-based assays using identical monoclonal antibodies (Figure 1). We found that the multiplexing QD-based gene expression study yielded the expected results at the single cell level where PSA is regulated transiently by the addition of R1881, and this effect can be blocked by the simultaneous presence of an anti-androgen Casodex (Figure 2).

There are several reasons why QD-based multiplexing technology could have unique roles in biology: 1) QD are bright and more resistant to photobleaching than the conventional organic fluorescent dyes; thus they are more efficient in detecting particularly low abundant target molecules in cells (Wu et al., 2003). 2) QD can be excited at a broad range of wavelengths but their emission spectra are specific with sharp and narrow peaks, dependent on their sizes and chemical compositions, with minimal overlap; thus they can be readily multiplexed and quantified (Gao et al., 2007). 3) QD can be modified chemically and linked to various targeting moieties for the detection and delivery of desirable molecules to cells and tissues (Gao et al., 2003, 2004). In our study, we confirmed that QD-based detection of AR and PSA is superior to conventional IHC and that they can be multiplexed for simultaneous detection of both AR and PSA in LNCaP cells. Ness et al. (2003) have shown that QD-based IHC staining for certain intracellular antigens in tissue sections was more sensitive than conventional IHC approaches (Ness et al., 2003). For example, ARCaP cells express only a low level of $A R$ and PSA (Zhau et al., 1996) which can be readily detected by QD-based IHC but not conventional IHC. While androgen-mediated PSA expression through AR has been studied extensively in LNCaP cells, none of the previous studies used multiplexing methods to detect the activation of $A R$, as evaluated by its nuclear translocation on a single cell basis, coupled with PSA expression. This multiplexed imaging of target genes allows investigators to identify multiple target genes of interest for: 1) elucidating complex cell-cell or protein-protein interactions; 2) clarifying spatial arrangements of cells within a tissue; 3 ) localizing multiple proteins within a cell; and 4) identifying complex protein expression patterns in single cells. Since the introduction of biocompatible QD in 1998, the potential of QD for multiplexing was quickly realized (Pinaud et al., 2006). However, actual demonstrations of this capability were only recently begun. Examples include preliminary two-color fluoroimmuno assay, cellular labeling and microbead and microsphere QD encoded bar codes used in DNA hybridization. In our work, we stained AR, PSA and EFl- $\alpha$ gene in cultured LNCaP cells using quantum dots with three colors $(655,605,565 \mathrm{~nm})$. The staining of all the markers was confirmed by spectra analysis. Although multiplexing can be obtained using quantum dots with different emitting wavelengths in cells or tissue specimens, the key future application of this assay is the quantification of different gene expressions. In this work, we developed a software program that can separate the different spectra from the composite data. We used the total area of each spectrum to reflect the gene expression level using a housekeeping gene EF- $1 \alpha$ as an internal control. The ratio between the target gene and internal control gene was generated and used for statistical analysis. Using this protocol, we confirmed the relationship between AR and PSA in single prostate cancer cells. QD-based multiplexing technology, once adopted by the scientific community, could address many important regulatory biologic questions in cells.

\section{Acknowledgements}

Supported by grants from the National Institutes of Health (P01 CA098912, R01 GM60652 and CA108468 to L.W.K.C., Department of Defense (17-03-2-0033 to L.W.K.C.), the Georgia Cancer Coalition Distinguished Cancer Scholar Awards (L.W.K.C.). This work was also partially supported by 973 program (N0. 2005CB522605), NFSC program (N0. 30400188), FANEDD award (NO. 200777) and IRT0712 to C.S. 


\section{References}

Alivisatos AP, Gu W, Larabell C. Quantum dots as cellular probes. Annu Rev Biomed Eng 2005;7:55-76.

Arya H, Kaul Z, Wadhwa R, Taira K, Hirano T, Kaul SC. Quantum dots in bio-imaging: Revolution by the small. Biochem Biophys Res Commun 2005;329:1173-7.

Chan P, Yuen T, Ruf F, Gonzalez-Maeso J, Sealfon SC. Method for multiplex cellular detection of $\mathrm{mRNAs}$ using quantum dot fluorescent in situ hybridization. Nucleic Acids Res 2005;33:161-8.

Chan WC, Nie S. Quantum dot bioconjugates for ultrasensitive nonisotopic detection. Science 1998;281:2016-8.

Gao X, Chung LW, Nie S. Quantum dots for in vivo molecular and cellular imaging. Methods Mol Biol 2007;374:135-46.

Gao X, Cui Y, Levenson RM, Chung LW, Nie S. In vivo cancer targeting and imaging with semiconductor quantum dots. Nat Biotechnol 2004;22:969-76.

Gao X, Nie S. Molecular profiling of single cells and tissue specimens with quantum dots. Trends Biotechnol 2003;21:371-3.

Gleave M, Hsieh JT, Gao CA, von Eschenbach AC, Chung LW. Acceleration of human prostate cancer growth in vivo by factors produced by prostate and bone fibroblasts. Cancer Res 1991;51:3753-61.

Huang WC, Wu D, Xie Z, Zhau HE, Nomura T, Zayzafoon M, et al. beta2-microglobulin is a signaling and growth-promoting factor for human prostate cancer bone metastasis. Cancer Res 2006;66:9108-16.

Kiyama S, Morrison K, Zellweger T, Akbari M, Cox M, Yu D, et al. Castration-induced increases in insulin-like growth factor-binding protein 2 promotes proliferation of androgen-independent human prostate LNCaP tumors. Cancer Res 2003;63:3575-84.

Michalet X, Pinaud FF, Bentolila LA, Tsay JM, Doose S, Li JJ, et al. Quantum dots for live cells, in vivo imaging, and diagnostics. Science 2005;307:538-44.

Mizokami A, Gotoh A, Yamada H, Keller ET, Matsumoto T. Tumor necrosis factor-alpha represses androgen sensitivity in the LNCaP prostate cancer cell line. J Urol 2000;164:800-5.

Ness JM, Akhtar RS, Latham CB, Roth KA. Combined tyramide signal amplification and quantum dots for sensitive and photostable immunofluorescence detection. J Histochem Cytochem 2003;51: 981-7.

Nomura T, Huang WC, Zhau HE, Wu D, Xie Z, Mimata H, et al. Beta2microglobulin promotes the growth of human renal cell carcinoma through the activation of the protein kinase A, cyclic AMP-responsive element-binding protein, and vascular endothelial growth factor axis. Clin Cancer Res 2006;12:7294-305.

Pinaud F, Michalet X, Bentolila LA, Tsay JM, Doose S, Li JJ, et al. Advances in fluorescence imaging with quantum dot bio-probes. Biomaterials 2006;27:1679-87.

Smith AM, Ruan G, Rhyner M N, Nie S. Engineering luminescent quantum dots for in vivo molecular and cellular imaging. Ann Biomed Eng 2006;34:3-14.

Thalmann GN, Sikes RA, Chang SM, Johnston DA, von Eschenbach AC, Chung LW. Suramin-induced decrease in prostate-specific antigen expression with no effect on tumor growth in the LNCaP model of human prostate cancer. J Natl Cancer Inst 1996;88:794-801.

West JL, Halas NJ. Engineered nanomaterials for biophotonics applications: improving sensing, imaging, and therapeutics. Annu Rev Biomed Eng 2003;5:285-92.

Wu D, Zhau HE, Huang WC, Iqbal S, Habib FK, Sartor 0, et al. cAMPresponsive element-binding protein regulates vascular endothelial growth factor expression: implication in human prostate cancer bone metastasis. Oncogene 2007;26:5070-7.

Wu X, Liu H, Liu J, Haley KN, Treadway JA, Larson JP, et al. Immunofluorescent labeling of cancer marker Her2 and other cellular targets with semiconductor quantum dots. Nat Biotechnol 2003;21:41-6.

Yeung F, Li X, Ellett J, Trapman J, Kao C, Chung LW. Regions of prostate-specific antigen (PSA) promoter confer androgen-independent expression of PSA in prostate cancer cells. J Biol Chem 2000;275:40846-55.

Yezhelyev MV, Gao X, Xing Y, Al-Hajj A, Nie S, O'Regan RM. Emerging use of nanoparticles in diagnosis and treatment of breast cancer. Lancet Oncol 2006;7:657-67.

Zhau HE, Chang SM, Chen BQ, Wang $Y$, Zhang $H$, Kao $C$, et al. Androgen-repressed phenotype in human prostate cancer. Proc Natl Acad Sci USA 1996;93:15152-7.

Zhou M, Ghosh I. Quantum dots and peptides: A bright future together. Biopolymers 2007;88:325-39. 
C. Shi et al. 